\title{
L-Dopa withdrawal in Parkinson's disease selectively impairs cognitive performance in tests sensitive to frontal lobe dysfunction
}

\author{
K.W. Lange ${ }^{1}$, T.W. Robbins ${ }^{2}$, C.D. Marsden ${ }^{1}$, M. James ${ }^{3}$, A.M. Owen ${ }^{2}$, and G.M. Paul ${ }^{1}$ \\ ${ }^{1}$ Department of Clinical Neurology, Institute of Neurology, ${ }^{3}$ Department of Psychology, The National Hospital for Neurology and \\ Neurosurgery, Queen Square, London, UK \\ ${ }^{2}$ Department of Experimental Psychology, University of Cambridge, Downing Street, Cambridge CB2 3EB, UK
}

Received June 17, 1991 / Final version September 17, 1991

\begin{abstract}
A group of ten patients with idiopathic Parkinson's disease (PD) was tested on a series of automated tests of learning, memory, planning and attention whilst either on or off L-dopa medication. Controlled withdrawal of L-dopa interfered with aspects of performance on three of the tests that had previously been shown to be sensitive to frontal lobe dysfunction; a spatial working memory task, the Tower of London planning test, and a visual discrimination paradigm that also included intra- and extra-dimensional shift tests of selective attention. More specifically, errors were increased in the spatial working memory test, and both the accuracy and latency of thinking were impaired. Thinking time was significantly slowed following L-dopa withdrawal, even though the possible contaminating effects on motor slowing were fully controlled by a yoked control procedure. Nine out of ten patients reached a further stage of the visual discrimination, set-shifting paradigm when on, rather than off, L-dopa medication. Spatial span was also impaired off medication, but there were no effects of L-dopa withdrawal on tests of pattern and spatial recognition memory, simultaneous and delayed matching to sample or visuospatial conditional associative learning. Comparisons with a large control group confirmed previous findings that PD is associated with deficits on the majority of these tests. The results are discussed in terms of the fronto-striatal, dopamine dependent nature of some of the cognitive deficits found in PD, but the apparent dopamine-independent nature of deficits in other aspects of cognitive functioning, notably in tests of visual recognition memory and associative learning.
\end{abstract}

Key words: Dopamine - Memory - Learning - Attention - Cognition - Parkinson's disease

L-Dopa has long been known to be efficacious in the treatment of the motor symptoms of idiopathic Parkin-

Offprint requests to: T.W. Robbins son's disease (PD) (Yahr 1990). However, it is becoming increasingly clear that patients with PD may also exhibit cognitive deficits, not only in late-in-the-course demented patients (for review see Lees 1990), but even in unmedicated PD patients, relatively early in the course of the disease (Lees and Smith 1983; Sahakian et al. 1988; Canavan et al. 1989; Downes et al. 1989; Cooper et al. 1991). Many of the deficits seen are reminiscent of those occurring after frontal lobe damage. For example, PD patients are impaired on the Wisconsin Card Sorting Test (WCST) (Lees and Smith 1983; see Brown and Marsden 1988, for a review), a classic index of frontal lobe dysfunction (Milner 1964), as well as in other forms of attentional set-shifting (Cools et al. 1984; Flowers and Robertson 1985; Downes et al. 1989).

What is less clear is the contribution of dopaminergic dysfunction to these frontal lobe like deficits. This contribution could occur in several distinct ways. For example, the striatum is intimately associated with the frontal lobes via corticostriato-cortical "loops" (Alexander et al. 1986) and damage to different sectors of the striatum can reproduce the effects of frontal lesions (Divac et al. 1967). Thus, it is possible that regional dopamine depletion from certain sectors of the striatum could lead to frontal lobe type deficits. In addition, the prefrontal cortex receives a direct mesencephalic dopaminergic innervation (Bjorklund and Lindvall 1984), and depletion of frontal dopamine in non-human primates produces impairments in delayed alternation that are reversible by treatment with L-dopa (Brozoski et al. 1979). In PD patients, the effects of $L$-dopa on performance on tests sensitive to frontal lobe dysfunction have been more difficult to define. In an early study, Bowen et al. (1975) found that L-dopa improved the number of categories attained on the WCST, without reducing the number of perseverative errors. More recently, Gotham et al. (1988) found variable effects of $\mathrm{L}$-dopa withdrawal in a group of PD patients. While verbal fluency was impaired off the drug, performance in two other tests, conditional associative learning and subject-ordered pointing appeared to show small improvements. Performance on the WCST was 
impaired both on and off $L$-dopa. From these results it could be concluded that L-dopa impairs, improves or has no effect upon cognitive function in PD.

Recently, we have able to define a number of deficits in PD in tests sensitive to frontal lobe damage in man, including the Tower of London test of planning (Shallice 1982; Owen et al. 1990a), a spatial working memory task (Owen et al. 1990a) and a test of attentional set-shifting (Downes et al. 1989; Owen et al. 1991). Although PD patients are impaired in each of these tests, the exact nature of the deficit differs from that seen in frontal lobe patients, and also depends upon the severity of the disease. For example, PD patients are slower to think about their solutions on the Tower of London problems, without necessarily being less accurate (Morris et al. 1988; Owen et al. 1990b), whereas frontal patients tend to be less accurate, but not slower in arriving at their solutions (Owen et al. 1990a). Therefore, we attempted to test PD patients both on and off $\mathrm{L}$-dopa medication in order to determine whether there were any consistent effects of L-dopa withdrawal.

PD patients, at least later in the course, also show impairments in aspects of visual memory that are not customarily associated with frontal lobe dysfunction, including delayed matching to sample, a test of visual recognition memory that has been used to define the neural substrates of visual memory in non-human primates (Mishkin 1982; Sahakian et al. 1988). The latter study showed delay-independent impairments in PD patients in a computerised matching to sample task which contrasted with the delay-dependent effects seen in patients with Alzheimer's disease. There were also deficits in tests of pattern and spatial recognition memory and in the learning of a paired associates pattern-location learning task. Therefore, we tested the psychological specificity of the effects of $\mathrm{L}$-dopa by comparing the effects of $\mathbf{L}$-dopa withdrawal on these tests of visual memory and learning with those seen on the tests of frontal lobe function described above.

\section{Materials and methods}

\section{Patients}

Ten patients with a diagnosis of idiopathic PD were studied after giving informed consent. All exhibited a typical bilateral akineticrigid syndrome with rest tremor. The patients had previously all been rated while on L-dopa according to the Hoehn and Yahr (1967) scale of clinical disability. Six of the patients were rated III, three were rated IV and one was given the most severe rating of disability, $V$. None of the patients showed clinical signs of depression, dementia, psychosis or focal cortical pathology. All of the subjects had been maintained on L-dopa preparations (Madopar and Sinemet) in combination with a peripheral decarboxylase inhibitor and had shown a considerable clinical response. The relevant patient data are presented in Table 1.

\section{Large control samples}

The main purpose of this study was to test the effects of L-dopa on cognitive function in PD patients on tasks already known to be

Table 1. Patient data (mean $\pm \mathrm{SEM})$

\begin{tabular}{|c|c|}
\hline Age & 4.9 years \\
\hline Sex & 2 females, 8 males \\
\hline Length of education & $9.8 \pm 0.4$ years \\
\hline Age at onset of disease & 5.3 years \\
\hline Duration of disease & 1.6 years \\
\hline Dosage of L-dopa & $1130 \pm 131 \mathrm{mg} / \mathrm{day}$ \\
\hline Duration of L-dopa therapy & $8.3 \pm 1.2$ years \\
\hline $\begin{array}{l}\text { Wechsler Adult Intelligence Scale } \\
\text { (verbal) }\end{array}$ & $113.2 \pm 3.6$ \\
\hline National Adult Reading Test & $115.6 \pm 2.6$ \\
\hline Kendrick Object Learning Test & $31.0 \pm 2.2$ \\
\hline
\end{tabular}

Table 2. Means and standard errors for normal control sample

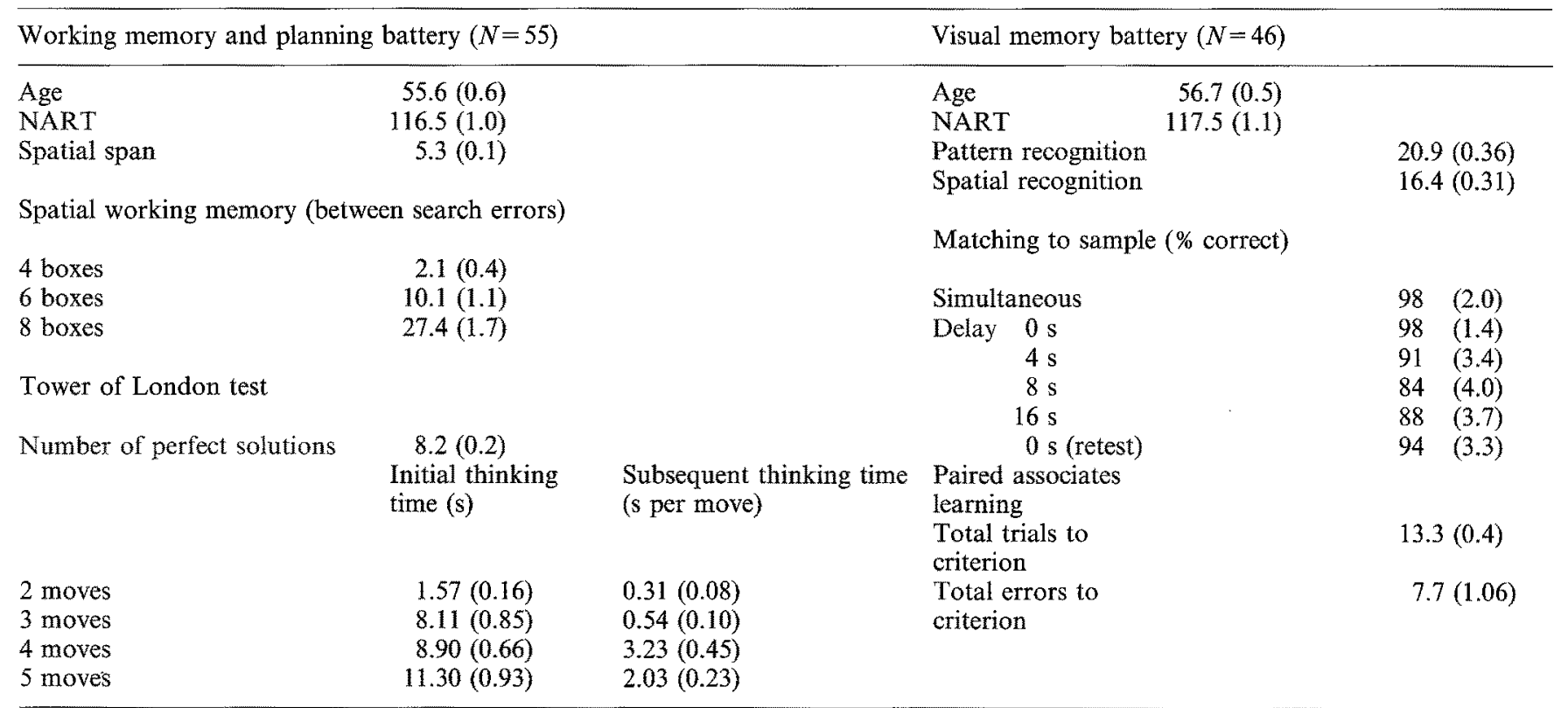


sensitive to deficits in PD. However, for the purpose of comparison, control data for the main dependent variables have been provided from the standardized scores of large groups of healthy normal control subjects and are shown in Table 2 . The control subjects were drawn from a large population of volunteers aged between 50 and 59 years from London, Cambridgeshire and Newcastle-upon-Tyne (North-East Age Research Panel). These controls had no history of neurological or psychiatric disorder.

\section{Procedure}

There were two testing sessions separated by a minimum of 4 and a maximum of 8 days. The order of testing on or off L-dopa was counterbalanced across the group; five patients were tested first on $\mathrm{L}$-dopa and five off $\mathrm{L}$-dopa, thus averaging possible practice effect across treatments. For the neuropsychological testing off L-dopa, the patients had ceased all antiparkinsonian medication for a minimum of $13 \mathrm{~h}$ prior to testing. The motor disability of each subject was assessed both on and off L-dopa using the King's College Hospital Parkinson's Disease Rating Scale. This evaluates 39 symptoms and signs each on a 0 to 3 scale with a maximum disability score of 117 .

\section{Clinical neuropsychological assessment}

Prior to the experiment, all patients received the National Adult Reading Test (NART) (Nelson 1982) to obtain an estimate of their optimal intellectual ability. Verbal IQ was prorated from two subtests of the Wechsler Adult Intelligence Scale (WAIS), Vocabulary and Similarities. The Kendrick Object Learning Test was also administered as a screen for possible dementia (Kendrick 1985).

\section{Experimental computerized tests}

The main testing procedures were taken from the Cambridge Neuropsychological Test Automated Battery (CANTAB), a series of computerized tests run on an Acorn BBC Master 128 Microcomputer, with a high resolution Microvitec 12 inch VDU and a Microvitec Touchtec 501 touch-sensitive screen. These were generally administered in one test session lasting for about $100 \mathrm{~min}$. Subjects were seated approximately $0.5 \mathrm{~m}$ from the monitor and it was explained that they would have to respond to stimuli by touching the screen.

They were introduced to the apparatus by way of a "sensorimotor screening task" in which they were asked to respond to a series of flashing crosses on the screen by placing the index finger of their preferred hand on the centre point of each cross. All subjects satisfactorily completed this task and so were allowed to proceed to the following experimental tests. The first three tests were then taken from the CANTAB Working Memory and Planning battery (see Owen et al. 1990a).

Spatial short term span. In this computerized version of the Corsi Block Tapping task, the ability of subjects to remember a sequence of squares highlighted on the screen was determined. Each trial began with the same arrangement of nine white $3 \mathrm{~cm}$ squares arranged quasi-randomly in the space of the screen. Subjects were instructed to observe the boxes, as some would change colour one after the other. Their task was to remember the location and the sequential order of the boxes changing colour. During each series, one box would change colour for $3 \mathrm{~s}$ and then return to white before the next in the sequence changed to the same colour. The subject was then prompted by a tone to repeat the sequence by touching the boxes in the same order. During this response sequence, each selected box changed to the same colour for one sec and a feedback tone sounded. Following one demonstration trial by the experimenter, the task began at the simplest level of difficulty with a two box sequence. After each successful trial, the number of boxes changing in the next sequence was increased by one to a maximum of nine boxes. After an incorrect attempt at any particular level, an alternative sequence of the same length was presented. This continued until the subject had failed three consecutive trials at any one level. During each trial, a number in the bottom left hand corner of the screen indicated the length of the current sequence. Also, all boxes changed to the same colour within each series, although on any two adjacent sequences different colours were used to minimize interference. The spatial short term memory span was calculated as the highest level at which the subject had successfully recalled at least one sequence of boxes.

The following three tests are all sensitive to frontal lobe damage (Owen et al. 1990a, 1991).

Spatial Working Memory Task. In this task the subject was required to "search through" a number of coloured $3 \mathrm{~cm}$ boxes presented on the screen, by touching each one in order to "open it", thus revealing its contents (see Fig. $1 A$ ). The colours of the boxes stayed constant within a trial, but varied over trials. The goal was to collect blue "tokens" hidden inside the boxes, and once found, to use them to fill an empty column at the side of the screen. The subjects were instructed that at any one time there would be a single token hidden
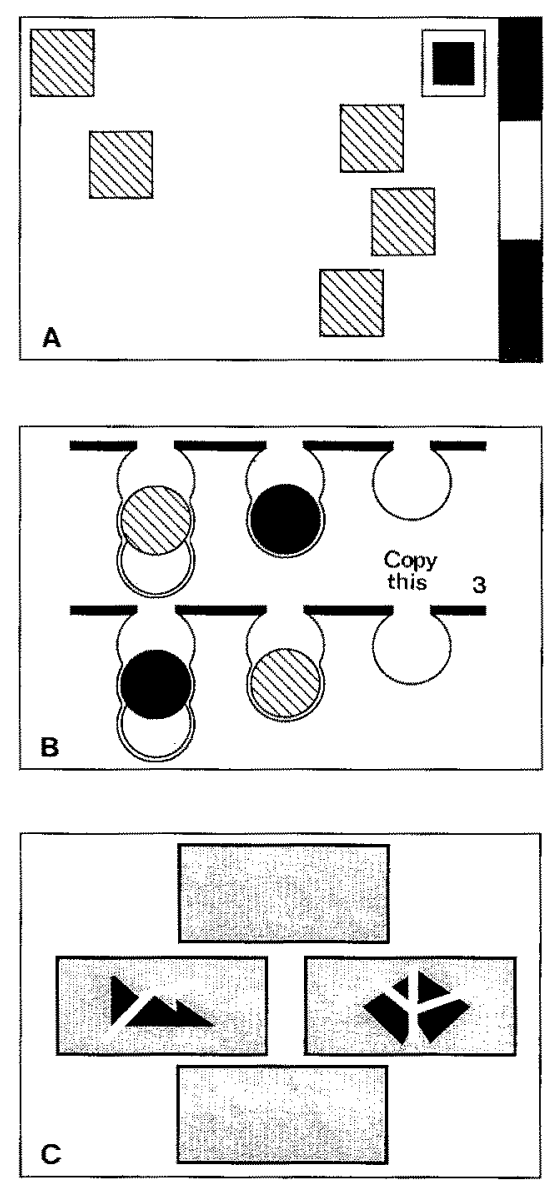

Fig. 1. A The arrangement of boxes on the screen for the spatial working memory task. The example shows a 6 box trial, in which one of the boxes has just been chosen to reveal a "blue token" which can now be added to three already in the column on the right hand side of the scren. This leaves the subject with 2 more tokens to find, in boxes in which they have not previously been located. B A sample 3 move problem for the modified Tower of London task. The goal position of the balls is shown in the top arrangement. $C$ Sample pairs of compound stimuli and their arrangement on the screen in the ID/ED attentional set shifting task 
inside one of the boxes. Their task was to search until they found it, at which point the next token would be hidden. The key instruction was that, once a blue token had been found within a particular box, then that box would not be used again to hide a token for that particular trial. Since every box was used once, on each trial the total number of blue tokens to be found corresponded to the number of boxes on the screen. In this task, two types of search error are possible. First, a subject may return to open a box in which a blue counter has already been found (a "between search" error). Second, a subject may return to a box already opened in the same search sequence (a "within search error"). Subjects could search the boxes in any order, but for control purposes, the number of boxes visited (excluding errors), before a token was found was determined by the computer. Thus, each subject received the same degree of feedback prior to the first error. After two practice trials with three boxes, there were four test trials with each of four, six and finally, eight boxes. The task was scored according to the number of between and within search errors at each level of difficulty, but the number of within search errors was sufficiently small to preclude analysis.

Planning task. This task is closely related to one developed by Shallice and McCarthy, the "Tower of London", that provides simpler versions of the "Tower of Hanoi" problem (Shallice 1982) Two sets of three coloured balls were presented, one in the top half of the screen and one in the bottom half. These were described to the subject as snooker balls since they appeared to be hanging in "pockets" or "socks" (see Fig. 1B). There were three pockets in each half of the screen, one that could clearly hold three balls, one that could hold two balls, and one that would be completely filled by one ball. On each trial a red ball, a blue ball, and a green ball were placed in predetermined positions in the pockets of each of the two displays. The subject was asked to rearrange the balls in the bottom display, such that their positions matched the "goal" arrangement in the top half of the screen. A ball could be moved by the subject first touching it and then touching an empty position in one of the other pockets. Once selected, a tone sounded and the rim of the ball began to flash, indicating that it was ready to be moved. At any time, the subject could cancel a selected ball by touching it a second time. "Illegal" moves, such as trying to remove a ball while there was another sitting above it in the same pocket, were carefully explained to the subject and, if attempted, they were registered, but evoked no response from the computer.

The starting position of the balls was varied such that in any particular trial the solution could only be reached after a minimum of two, three, four or five moves. Subjects were instructed to examine the position of the balls at the beginning of each problem and attempt to solve it in the minimum possible number of moves. This was both given to them verbally and displayed on the screen throughout each trial. They were encouraged not to make the first move until they were confident that they could execute the entire sequence needed to solve the problem. The maximum moves allowed corresponded to twice the minimum number possible plus one, or plus two, in the case of five move problems. Following the successful completion of each problem, the VDU displayed the word "FINISHED", before moving on to the next trial. The program stored the number of moves required by the subject to rearrange the balls and measured the selection and movement latencies for both the first and the subsequent moves. After six practice problems with one and two moves, the subject was given two each of two and three move problems and four each of four and five move problems. These test problems corresponded exactly to those used in the original Tower of London test.

For each test problem a "yoked control" condition was employed to provide baseline measures of motor initiation and execution times. On each trial of this control condition, the subject was required to follow a sequence of single moves executed by the computer in the top half of the screen by moving the corresponding ball in the lower arrangement. Thus, initially the two arrangements differed by just one ball. Once the subject had made the appropriate move, the top arrangement changed again, so that the subject had to make another single move. The test was "yoked" to the main test in the sense that in each trial, the movement of the balls was an exact replication of those moved by the subject in the corresponding test trial. The measurement of selection and execution latencies in this control condition provided baseline estimates of motor initiation and execution times.

Test trials and "yoked" control trials were arranged in four blocks of six problems each. The first six test trials were given (two problems at each of two, three and four moves), followed by their corresponding "yoked" control trials. Then the remaining six test problems were presented (two at four moves and four at five moves), followed by their yoked control trials. Between each block change there were two practice trials to ensure that the requirements of the current set of tasks had been fully understood.

Accuracy of performance. Across the 12 test problems the main measure was the proportion of problems solved in the minimum number of moves specified ("perfect solutions").

Latency measures of performance. Baseline measures of motor initiation and motor execution times were extracted from the 12 "yoked control" trials. In all cases, latencies were recorded in centiseconds and converted to seconds for the purpose of presentation. The motor initiation time was the mean time between the onset of each problem, and the initiation of the first move (i.e. a correct touch of the required ball). The motor execution time was the time between touching the first ball and completing the sequence of single moves that comprise the whole problem. Since these control problems were yoked to the test problems, the total execution time was divided by the number of moves to provide an estimate of the average movement time per move. The motor initiation and execution times were used to derive estimates of planning or "thinking" time in the main task. Two separate estimates were calculated. In each problem, the initial thinking time was the time between the presentation of the problem and the first touch, minus the corresponding motor initiation time, as calculated from the "yoked control' task. The subsequent thinking time was the time between the selection of the first ball and the completion of the problem minus the total motor execution time from the corresponding control problem. Since this measure clearly varied with problem length, subsequent thinking time scores were divided by the number of moves to give an estimate of the average thinking time "per move". In this way, pure estimates of initial and subsequent thinking times were derived, unconfounded by motor initiation or execution times.

Visual discrimination paradigm; attentional set-shifting. The next test to be administered was taken from the CANTAB Attentional test battery.

The test set-up and stimuli were shown to the subjects. Four rectangular boxes, to the top and bottom and to the right and left of centre, appeared on the screen. Two of these contained the test stimuli but the boxes used changed from trial to trial. Subjects were instructed in the following way:

Now you can see two patterns. One of the patterns is correct. You must point to the one you think is correct. There is a rule you can follow to make sure you make the correct choice each time. The computer will be keeping track of how well you are doing and when it is clear that you know the rule the computer will change it, but this will not happen very often. To begin with, there is nothing on the screen to tell you which of the two patterns is correct, so your first choice will be a simple guess. However, the computer will give a message after each attempt to tell you whether you are right or wrong. You can start now.

The test then proceeded through a number of stages, each with a different contingency, up to a maximum of nine (see Fig. $/ \mathrm{C}$ ). For each, continuation to the next stage was dependent on a criterion of six successive correct discriminations being reached. If criterion was not reached by the 50th trial of a stage, then the test was discontinued and subjects did not proceed to the following stage. The order of discrimination was fixed, so that the EDS always 
followed the IDS. However, previous work has established that comparable effects are found when the alternative ordering is used (Roberts et al. 1988). More detailed rationales for the exact design of the test can be found in previously published articles (Downes et al. 1989; Roberts et al. 1988).

To begin with, subjects were given a simple simultaneous discrimination in which the stimuli varied along only one of the two "dimensions" for deriving the stimuli. These dimensions were purple-filled shapes or white lines. The starting dimension was balanced across subjects and groups. A response to one of the two boxes containing the stimuli resulted in an auditory tone, together with visual feedback which informed the subjects of the correctness of their responses. This feedback was in the form of the words "correct" and "wrong" presented, respectively, in green and red lettering above the middle two boxes. The same feedback was used for each of the subsequent stages. After $1.5 \mathrm{~s}$ the screen cleared and an intertrial interval of $1 \mathrm{~s}$ was begun.

Following the initial simple discrimination (SD), the remaining eight stages were as follows. For the second stage (SDR), the discriminanda remained the same, but the previously incorrect choice became the correct one and vice versa, that is the contingencies were reversed. At the third stage (C-D) the second dimension was introduced with one exemplar of each dimension paired together to form a compound stimulus in two of the response boxes. To succeed, a subject had to continue to respond to the correct exemplar of the previous stage. For this and all subsequent stages, exemplars of the different dimensions were paired in a pseudorandom fashion so that all four possible compound stimuli were used, with the constraint that runs of no more than three trials with the same pairings were allowed. The stimuli for the fourth stage (CD) and subsequent stages were also compounds, but the two exemplars from the different dimensions were superimposed, with the white line always in the foreground. The contingencies were again unchanged from those for the previous two stages. A reversal then occurred at the fifth stage (CDR). New exemplars for both dimensions were introduced at the sixth stage, the intra-dimensional shift (IDS), but the relevant dimension (i.e. shapes or lines) was unchanged from stage 1 . This was followed by a further reversal at the seventh stage (IDR). For the penultimate stage, the extra-dimensional shift (EDS), new exemplars were again introduced, but success at this point depended on the subject shifting response set to the exemplars of the previously irrelevant dimension. Finally, contingencies were reversed to the previously incorrect exemplar of the new dimension (EDR). The main measure of performance on this task was the stage successfully attained.

Tests of visual memory and learning. In the last part of the test session, all patients received computerized tests of visual memory and learning identical to those previously described in detail (Sahakian et al. 1988). These included tests of pattern and spatial recognition, simultaneous and delayed matching to sample, and a conditional visuospatial associative learning task. In the pattern recognition task, a number of abstract visual stimuli are displayed one by one in the centre of the screen before recognition memory is tested in a two-alternative forced choice task, where the original stimulus is presented with a novel one and the subject has to pick the former. The test consists of two "lists" of 12 stimuli each, comprising 24 in all. In the spatial recognition task, open white squares are presented in a number of spatial locations on the screen, before recoguition memory for location is tested, again with a two-alternative forced choice procedure. There were 4 blocks of 5 stimuli each, comprising 20 in all. In the matching to sample test, the first phase (perceptual matching) consists of the simultaneous presentation of a complex abstract stimulus, varying in both colour and shape, with four matching stimuli. Subjects are required to choose the matching stimulus in a four-alternative forced choice procedure. In the second phase (memory matching), the matching stimuli are presented in five trial blocks following a delay of 0,4 , 8 or $16 \mathrm{~s}$, followed finally by a retest at $0 \mathrm{~s}$. This delayed matching to sample test thus assesses the forgetting of the stimuli to be recognised after a short delay.
The conditional visuospatial paired associates test is identical to one described as the delayed response task in Sahakian et al. (1988). Here, however, the emphasis was on the learning as distinct from mnemonic aspects of the task. Abstract visual stimuli were presented one by one in a ring of six (and in the last stage, eight) "boxes" arranged around the screen. The stimuli were then re-presented one by one in the centre of the screen and the subject indicated in which box they had been previously presented. Subjects were given up to ten trials to learn the location of each stimulus of the set, before proceeding to the next stage. Failure to reach criterion at any stage resulted in the premature termination of the test. The test began with only one stimulus location to be remembered and then gradually increased to $2,3,6$ and 8 stimuli.

\section{Statistics}

The main hypothesis to be tested was the effects of L-dopa on performance in the PD group and this was generally assessed in a within-subjects design using analysis of variance (Winer 1971) with one or two factors depending on the task.

\section{Results}

\section{Effects of L-dopa on motor disability score}

As expected, L-dopa withdrawal led to considerable worsening of ratings of motor disability (mean scores, on L-dopa $16.8 \pm 2.0$; off L-dopa $63.9 \pm 4.4$ ).

\section{Comparison of cognitive performance of $P D$ patients and controls}

Table 2 summarizes mean ( $\pm S D$ ) scores for most of the dependent variables used in the cognitive test battery for large groups of healthy control subjects of similar age and verbal IQ to the PD group used in this study. Comparisons of these values with those shown by the present PD patients confirm the results of earlier studies (Sahakian et al. 1988; Morris et al. 1988; Owen et al. 1990b) showing substantial deficits in the medicated PD group in each of the tests of visual memory (pattern and spatial recognition, delayed matching to sample) or learning (conditional visuospatial associative learning) and two of the tests sensitive to frontal lobe dysfunction (the Tower of London (for initial thinking time only) and attentional set-shifting). In the cases of spatial working memory and spatial span, there were no significant deficits in this group of PD patients on L-dopa medication, again consistent with previous findings (Morris et al. 1988).

\section{Effects of $\mathrm{L}$-dopa medication on tests sensitive to frontal lobe dysfunction}

Tower of London planning task; thinking latency and accuracy. Figure 2 shows the performance of the PD group on and off L-dopa medication for measures of the accuracy and latency of thinking in the Tower of London planning task. In general, patients performed significantly better on L-dopa for both the accuracy and latency variables. As expected, initial thinking time (Fig. 2A) was longer as a function of problem difficulty $(F=11.90$, $d f=3,27 ; P<0.001)$. There was a significant lengthening 


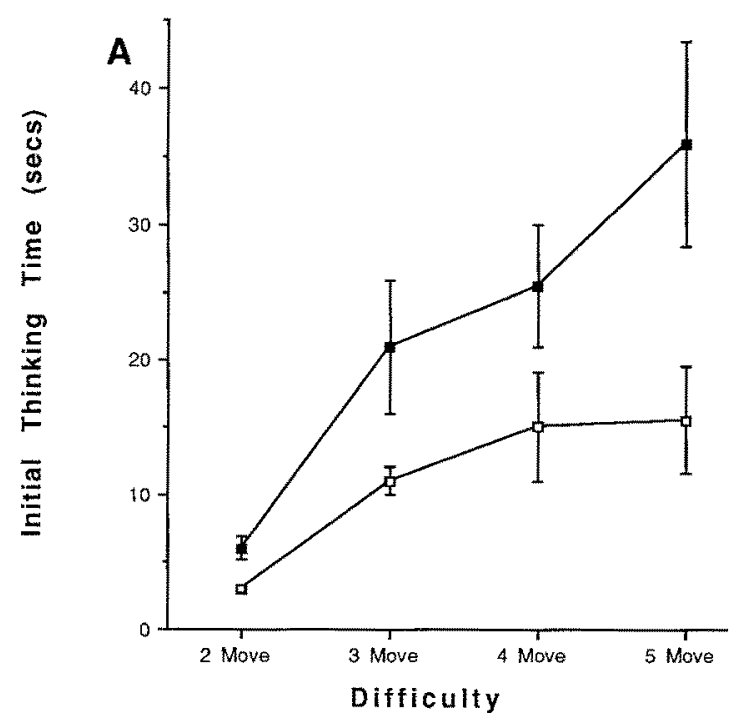

Fig. 2A, B. Performance of patients with Parkinson's disease on $(-\square-)$ or off L-dopa (-) medication on the Tower of London test of planning: A initial thinking time; $\mathbf{B}$ accuracy of solutions

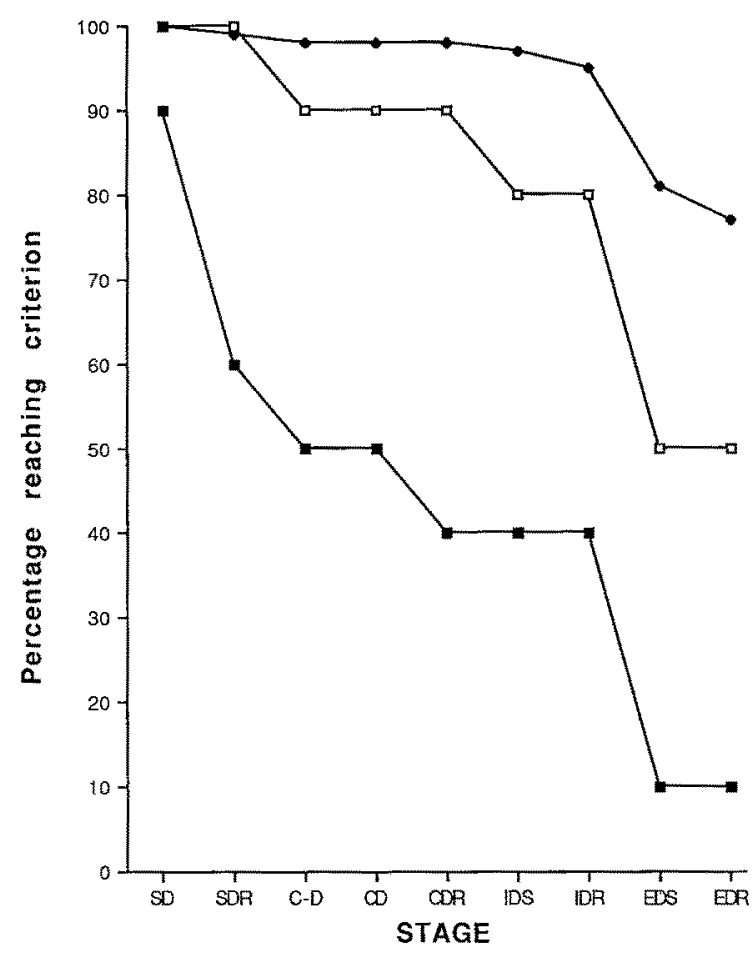

Fig. 3. Performance of patients with Parkinson's disease on the Visual Discrimination, set shifting paradigm, either on or off $\mathrm{L}$-dopa medication. Ordinate: proportion of subjects reaching the learning criterion of $6 / 6$ within 50 trials. Abscissa: stage of discrimination learning; $S D=$ simple discrimination, $S D R=$ simple reversal; $C-D=$ compound discrimination; $C D=$ compound discrimination (superimposed dimensions); $C D R=$ compound discrimination reversal; $I D S=$ intradimensional shift; $I D R=$ intradimensional reversal; $E D S=$ extradimensional shift; $E D R=$ extradimensional reversal. For further explanation, see Materials and methods. $(-\square-)$ On L-dopa; $(-\mathbf{-}-)$ off L-dopa; $(-\rightarrow)$ controls, $(n=129)$

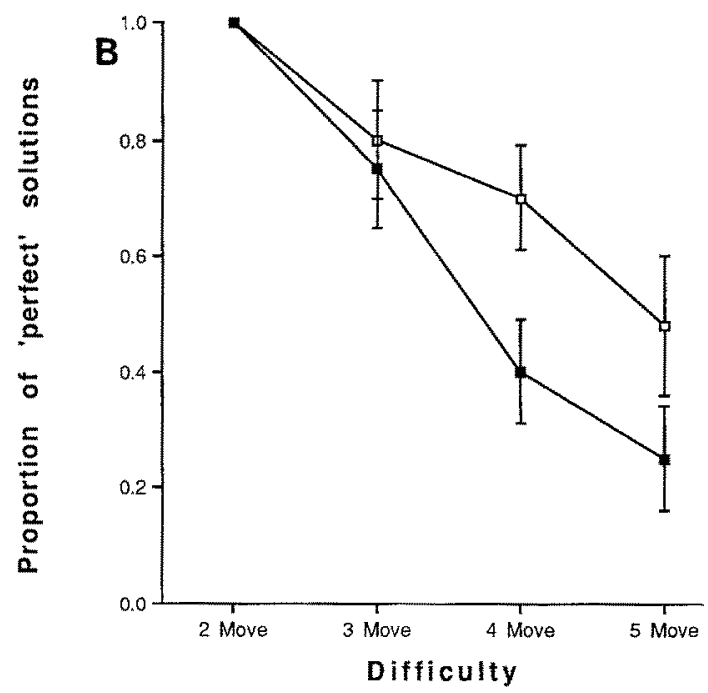

(proportion of perfect solutions) at each level of difficulty. The vertical bars indicate $\mathrm{SEM}$ values

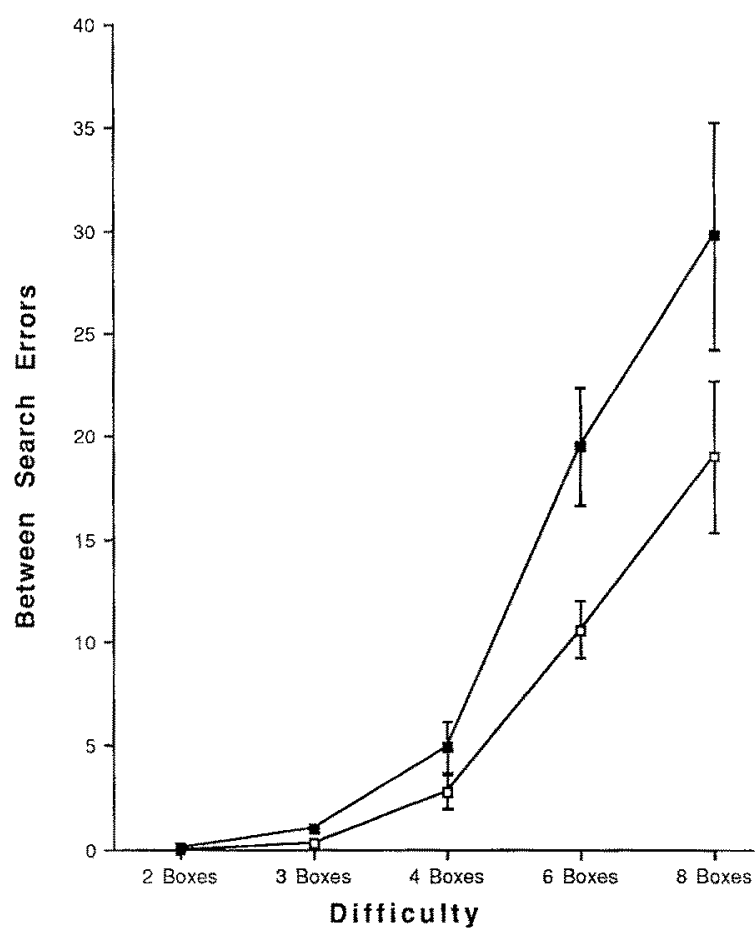

Fig. 4. Performance of patients with Parkinson's disease either on (- $\square-$ ) or off $\mathrm{L}$-dopa (- medication on the spatial working memory test. The mean number of between search errors is shown at different levels of task difficulty. The vertical bars indicate SEM values

of initial thinking time when patients were tested off L-dopa $(F=16.79, d f=1,9 ; P<0.001)$ and this effect depended to some degree on the level of difficulty $(F=3.02$, $d f=3,27 ; P<0.05)$. The initial thinking time shown in the medicated phase was comparable to that seen in previous studies (Morris et al. 1988; Owen et al. 1990b), averaging about 15 seconds at the more difficult ( 4 and 


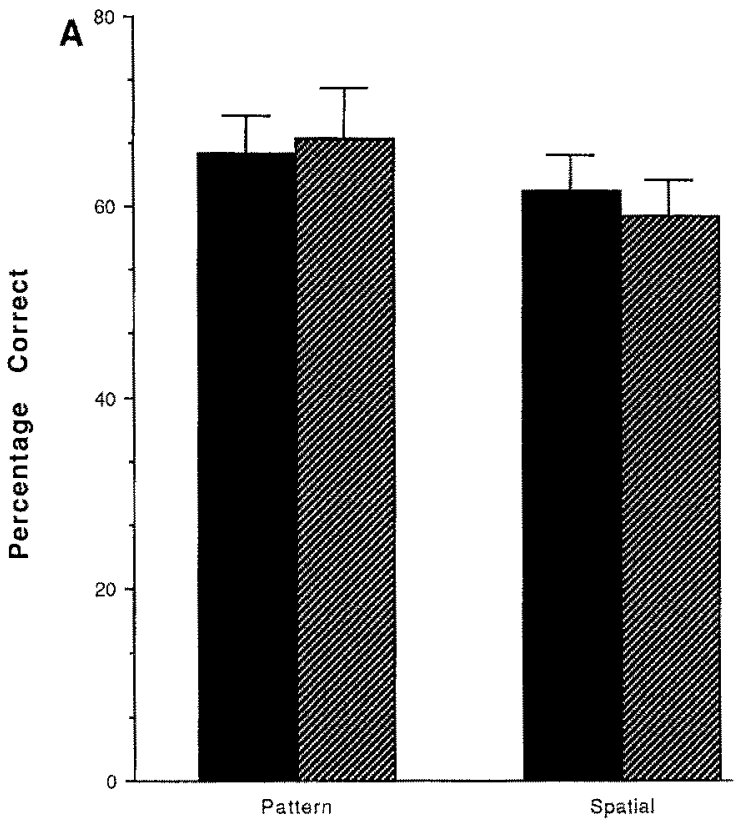

TEST

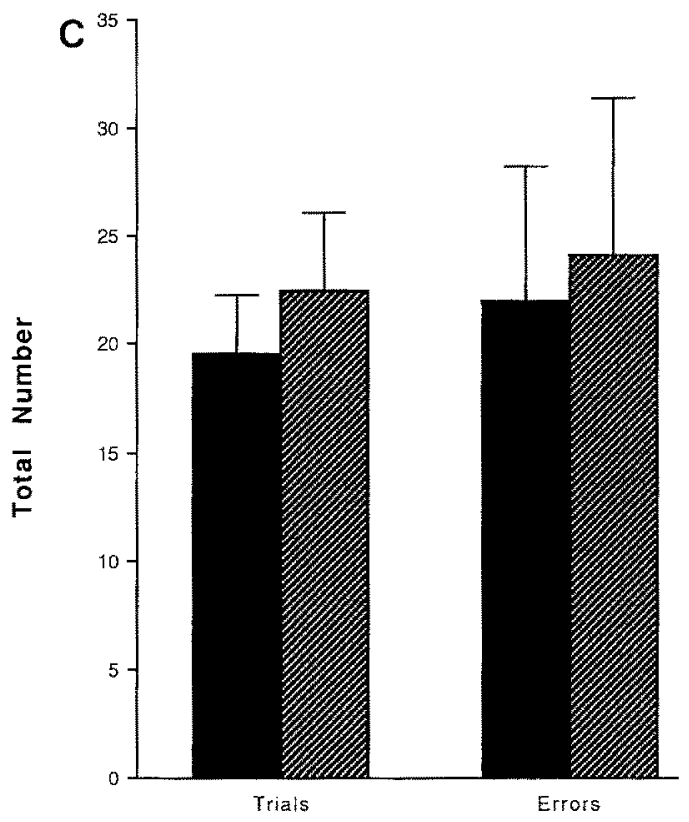

Measure

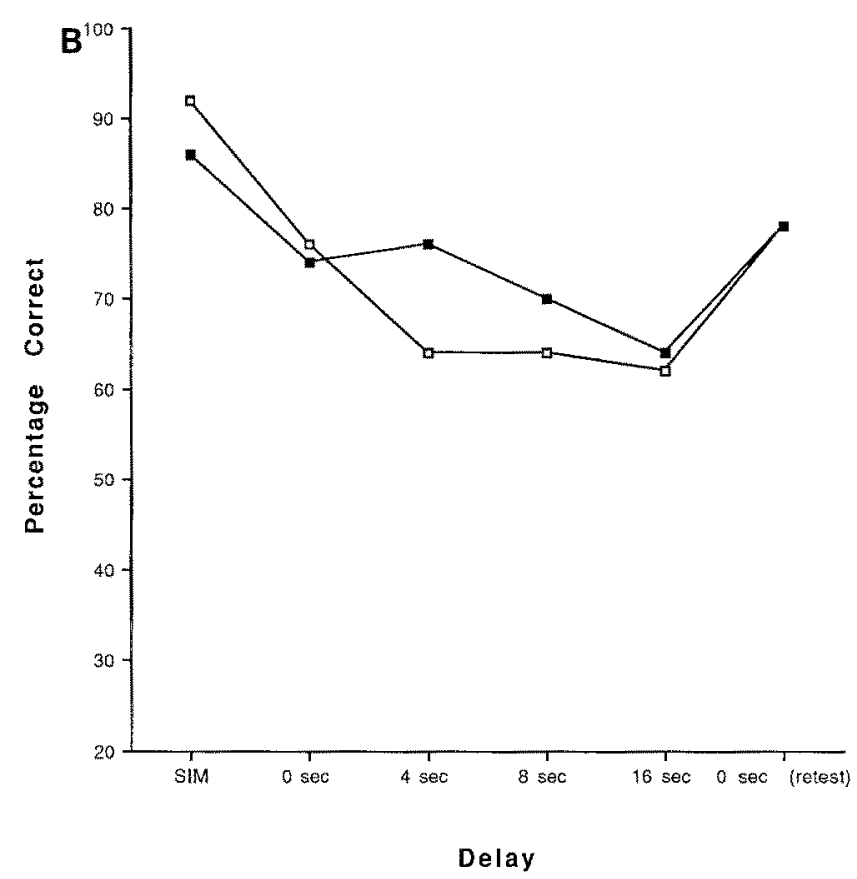

Fig, 5A-C. Lack of effect of L-dopa withdrawal on performance of three tests of visual memory and learning: A Pattern and spatial recognition memory ( $\boldsymbol{\square}$ ) On L-dopa; (ख्य) off L-dopa; B matching to sample performance (both simultaneous and delay components) $(-\square)$ On L-dopa; $(--)$ off L-dopa; $\mathbf{C}$ conditional visuospatial associative learning ( $\boldsymbol{\square})$ On L-dopa; (浮) off $\mathrm{L}$-dopa. Shown on the left is mean cumulative trials to reach criterion up to and including the set of 8 stimuli. Shown on the right is the mean number of cumulative errors committed in attaining criterion up to and including the set of 6 stimuli. The vertical bars indicate SEM values
5 move) problems. Following L-dopa withdrawal, the initial thinking time increased substantially to over $35 \mathrm{~s}$.

Compared with normal controls (Table 2) prolonged subsequent thinking times in the PD patients were not evident when patients were on L-dopa (mean scores, 0.26 , $1.03,2.12$ and $1.92 \mathrm{~s}$ per move at $2,3,4$ and 5 move problems, respectively). However, withdrawal of L-dopa significantly lengthened this index of thinking latency $(F=5.15, d f=1,9 ; P<0.05)$, the respective mean durations at each level of problem difficulty being $0.51,1.10$, 3.00 and $2.78 \mathrm{~s}$ per move. There was also a main effect of difficulty $(F=9.66 ; d f=3,27 ; P<0.01)$, but no interaction with L-dopa medication $(F<1.0)$.

Thinking accuracy, as measured by the most sensitive index, perfect solutions (i.e. those solved in the minimum number of moves), also showed an impairment when tested off L-dopa medication (Fig. 2B), but only for the 4 and 5 move problems $(F=15.3, d f=1,9, P<0.01)$. However, performance of the PD group on medication was comparable to that of the large control sample (Table 2), averaging about $8 / 12$ problems solved in the minimum number of moves in both cases. 
Tower of London; yoked motor control measures. On the yoked motor control test, the PD patients were unaffected by L-dopa withdrawal either for the initial or the subsequent movement time measures (both $F<1.0, \mathrm{NS}$ ). The mean values for the initial movement time were $2.14 \mathrm{~s}$ (off L-dopa) and $2.25 \mathrm{~s}$ (on L-dopa). For subsequent movement time, the equivalent values were $3.2 \mathrm{~s}$ and $2.8 \mathrm{~s}$.

Visual discrimination paradigm; attentional set-shifting. Figure 3 shows the proportion of PD patients reaching criterion at each stage of the discrimination learning paradigm. The performance of the patients on L-dopa was strikingly similar to that of the medicated PD patients described by Downes et al. (1989). For comparison, the performance of a group of 129 healthy controls is shown, confirming the deficit shown previously in PD (Downes et al. 1989). Only $50 \%$ of the patients on L-dopa successfully completed all stages of the task, compared to the control rate of about $74 \%$. Most of the failure was at the attentional set-shifting stage (i.e. the extradimensional shift), where $38 \%$ of those PD patients attempting this stage failed, compared to the control value of $17 \%$. As shown in Fig. 3 performance off $L$-dopa medication was significantly worse than on the drug. Nine out of ten PD patients reached a further stage of the test on L-dopa (binomial test, $P=0.022$, two-tailed).

Spatial working memory. Figure 4 shows the effects of L-dopa on between search errors, the main index of performance on the spatial working memory task. There was a significant Difficulty $\mathrm{X}$ Medication interaction $(F=18.03, d f=4,36 ; P<0.001)$, with performance on $L$-dopa being superior at the six and eight box levels.

\section{Effects of $\mathrm{L} \sim$ dopa medication on spatial span and visual memory}

The PD patients had a span of $4.4( \pm 0.3)$ on $L$-dopa and $3.2( \pm 0.2)$ off the drug, a significant difference $(F=17.05$, $d f=1,9 ; P<0.001$ ). Normal controls average 5.3 (Table 2).

Figure $5 \mathrm{~A}$ shows that spatial and pattern recognition performance were not at all affected by L-dopa medication (both $F<1.0, \mathrm{~ns}$ ), although the overall performance of the PD patients was markedly deficient compared with the control sample (Table 2).

Figure 5B shows that L-dopa had no beneficial effect on matching to sample performance, either for the simultaneous or the delayed versions $(F=1.33, d f=3,27$; NS), although comparison with the data of Table 2 shows the delay-independent deficit in matching to sample performance originally described by Sahakian et al. (1988).

Latency measures on the matching to sample task were unaffected by L-dopa medication. For simultaneous matching to sample the PD patients averaged $5.2 \mathrm{~s}$ on L-dopa and $4.7 \mathrm{~s}$ off the drug $(F<1.0, \mathrm{~ns})$. For delayed matching to sample the latencies on the drug varied from $4.3 \mathrm{~s}(0 \mathrm{~s}$ delay) to $4.98 \mathrm{~s}(4 \mathrm{~s})$, whereas off $\mathrm{L}$-dopa the scores were slightly, but not significantly $(F=2.03$, $d f=1,9)$, increased to $4.44 \mathrm{~s}(0 \mathrm{~s}$ delay) to a maximum score of $6.02 \mathrm{~s}(16 \mathrm{~s})$.

Conditional visuospatial associative learning. Whereas healthy subjects hardly ever fail this task (Sahakian et al. 1988), seven out of ten PD patients successfully reached the criterion of learning at the most difficult level (eight boxes) on this task when on L-dopa, while $6 / 10$ patients were successful off the drug. Using a more sensitive trials to criterion index, Fig. 5C shows that L-dopa had no substantial effects on the trials to criterion measure of this learning test $(F<1.0, \mathrm{~ns})$ even though the PD patients were performing at a markedly inferior level to that of healthy controls (Table 2), in confirmation of earlier findings (Sahakian et al. 1988). Also shown on Fig. 5C is the lack of effect of L-dopa on errors to criterion, only including the data for six boxes, which all patients attempted, both on and off L-dopa; again there were no significant effects of medication $(F<1.0, \mathrm{~ns})$.

\section{Discussion}

The main results found in this study were that L-dopa withdrawal selectively impaired performance on some of the tests of cognitive function, notably those previously shown to be sensitive to frontal lobe damage, without affecting performance on other tests of visual memory and learning. These results suggest that the effects of L-dopa are psychologically specific in some sense and argue that some, but not all, of the parkinsonian cognitive impairment is due to a loss of central dopaminergic function.

The PD patients were impaired in all three of the tests previously shown to be sensitive to frontal lobe dysfunction. On the Tower of London test, the initial thinking time was slower than normal, as previously documented (e.g. Morris et al. 1988; Owen et al. 1990b). However, L-dopa withdrawal dramatically exacerbated this deficit and led to average thinking times of about $36 \mathrm{~s}$ on the most difficult (five move) problems. Two other measures on this test also showed large effects of L-dopa treatment, although these aspects of the test are not generally impaired in patients on medication. Subsequent thinking time (i.e. the time taken to think about the problems subsequent to the first move) was also lengthened in the absence of L-dopa, and the number of perfect solutions of the problems, an index of thinking accuracy, also declined under these conditions. These latter results are of particular interest as they suggest that L-dopa therapy may normally be masking underlying deficits, which become apparent upon its withdrawal. A similar conclusion holds for the test of spatial working memory, where the PD patients exhibited no deficits in terms of the number of between search errors (as in previous studies, e.g. Morris et al. 1988) but L-dopa withdrawal led to substantial impairment. These findings may also help to explain why there is an apparent progressive impairment on some of these tests in PD patients. Thus, it has recently been found that PD patients with severe clinical disability can show increased numbers of errors on the spatial 
working memory test, as well as impaired thinking accuracy on the Tower of London test, even when on medication (Owen et al. 1990b). It is possible for these patients either that $L$-dopa is losing some of its efficacy in counteracting the cognitive impairments or that its effects are being undermined by the progressive loss of central dopamine in frontostriatal regions.

Probably the most sensitive of the three frontal tests to PD is the attentional set-shifting task, particularly in its extradimensional shift phase. A previous study has found that unmedicated, early-in-the-course PD patients are impaired in this task (Downes et al. 1989), whereas their performance on the Tower of London and the spatial working memory task is essentially normal (Owen et al. 1990b). It is noteworthy that the former study found that, if anything, the unmedicated PD patients were more severely impaired than the later-in-the course medicated PD patients (who are generally more cognitively impaired on other tests, see Sahakian et al. 1988). This suggests that the $L$-dopa medication may have been promoting effective attentional shifting in these patients, thus boosting their performance even above that of the unmedicated, mildly affected patients. Some evidence for this possibility was obtained in the present study. Nine out of ten of the PD patients reached a later stage in the discrimination learning paradigm when under $\mathrm{L}$-dopa medication. These data support the possibility that striatal or frontal dopamine may influence attentional set. However, the PD patients were so impaired on this test when off medication, that very few of them actually reached the stage of extradimensional shifting and thus it was difficult to test the specific hypothesis that dopamine is particularly important for attentional shifting per se.

The results for the discrimination learning paradigm are in ageement with those of Bowen et al. (1985), who found that patients under L-dopa achieved more categories on the WCST. They are less consistent with those of Gotham et al. (1988) or an unpublished study by Gotham, Brown and Marsden showing no detrimental effect of L-dopa withdrawal on WCST performance. The reasons for this discrepancy may arise from subtle taskrelated factors. For example, the WCST is a more complex test than the attentional set-shifting paradigm and the PD patients may fail it for reasons unrelated to their failure on the discrimination learning paradigm. It is also possible that the effects of L-dopa withdrawal in Parkinson's disease vary according to the stage of the disease. As can be seen from the Hoehn and Yahr ratings of clinical disability, the present PD patients were quite severely affected, and so were presumably quite late in the course of the disease. It is possible that L-dopa medication is disruptive rather than beneficial to cognition in patients earlier in the course of the disease and this possibility requires further evaluation.

In general, the results we have found are apparently consistent with those of Gotham et al. (1988) for verbal fluency, another commonly used frontal lobe test. However, they do not lend support to the hypothesis that frontal lobe type deficits arise from L-dopa medication, as suggested from the results of that study. This hypoth- esis suggests ingeniously that the caudate nucleus is "overdosed" with L-dopa medication as a result of the optimal treatment of the motor deficits that arise from the more severe loss of dopamine in the putamen. The main data in support of this position are the results of two tests, conditional associative learning and subject ordered pointing, which are both suggested to be sensitive to frontal lobe damage on the basis of other work (see Milner 1982). However, in relation to the present results, the following points can be made. First, although the test of conditional associative learning used in the study by Gotham et al. (1988) is sensitive to the effects of frontal lobe damage (Canavan et al. 1989), its strong learning and memory components may also make it susceptible to other forms of CNS damage, for example in the right hippocampus (Petrides 1985). Second, a test of conditional associative learning was also used in the present study, but L-dopa withdrawal had no effect (see below). Third, close examination of the Gotham et al. results suggests in fact that there is no discrepancy with the present results. When a direct comparison was made between the effects of the presence and absence of $L$ dopa, no significant effects in either conditional learning or subject ordered pointing were present. The apparent deficit in the L-dopa treated condition arose in comparison with an untreated control group.

The present results could conceivably arise from some form of artifact produced by L-dopa withdrawal, but we think that this is unlikely. First, it could be argued that the effects are secondary to the imposition of severe parkinsonian motor symptoms, which may have the effect of distracting the patient from task requirments or more directly affecting his or her responding to the touch sensitive screen. It is certainly the case that the clinical measures of motor function revealed a significant effect of $L$-dopa withdrawal. However, it is unlikely that they contributed materially to the observed cognitive deficits for the following reasons. First, on the Tower of London, any motor effect of the disease was taken into account when correcting for thinking times. Thus, patients were required to make moves in a yoked situation which required no planning and these scores were used to adjust the actual thinking times. Second, and perhaps more importantly, there were no significant effects of L-dopa removal on the latency to respond, either in the yoked control task for the Tower of London, or on other measures of latency, for example, on the matching to sample task. Another possible explanation of the impairment produced by L-dopa withdrawal is an indirect result of depressed mood and arousal (Brown et al, 1984). However, in a later study, Gotham et al. (1988) found no relationship between changes in mood and arousal and task performance. In this study, we argue against this explanation because the pattern of cognitive improvement under L-dopa was specific. Thus, visual memory and learning were not impaired on L-dopa withdrawal, and there seem to be no obvious ways in which these tasks would benefit differentially from elevations in mood or arousal. Indeed, a recent study has shown that the visual memory and learning tests used here are sensitive to deficits in elderly depressed individuals (Abas et 
al. 1990). It is also worth noting that the motor requirment of, for example, the attentional set shifting task, is not substantially different from that of the delayed matching to sample procedure.

The PD patients tested here, while not demented, were quite seriously impaired in all of the tests of visual memory and learning, thus supporting previous work (Sahakian et al. 1988; El-Alwar et al. 1987; Brown and Marsden 1988 for a review). Quite surprisingly, these deficits were not at all responsive to L-dopa withdrawal. This can hardly be due to differences in task sensitivity, as the tests of visual memory and learning are more sensitive to early in the course Alzheimer disease than the attentional setshifting task (Sahakian et al. 1990). Nor is it because of any lack of consistency in these measures or patient selection, as other authors have found exactly the same result for these same memory tests in a different group of PD patients (Gotham, Brown and Marsden unpublished results). Thus, these impairments in memory function may be mediated by non-dopaminergic mechanisms, perhaps consistent with the wealth of evidence that PD patients may have deficits in the functioning of the cholinergic, noradrenergic and serotoninergic innervations of the cortex and the hippocampus (Agid et al. 1987). It is perhaps surprising that L-dopa did not modulate memory function in view of the suggestion that dopamine contributes to the "effortful" processes of free recall that are often deficient in patients with PD and depression (Weingartner et al. 1984). However, this may not apply to the present tests, which mainly employed tests of memory function based on recognition rather than recall.

The only result which does not support our strong argument of a selective effect on frontal lobe type functions is on the test of spatial span, which did show large effects of $\mathrm{L}$-dopa removal, but is not very sensitive to the effects of frontal lobe damage (Owen et al. 1990a). One possible explanation for this is that the Corsi span task might be sensitive to the effects of damage of other cortical regions, for example, the right parietal cortex, which has recently been shown to among those more posterior cortical regions that receive a substantial dopaminergic innervation in the primate brain (Berger et al. 1991).

Overall, we believe that we have demonstrated profound deficits in tests sensitive to frontal lobe damage in PD patients experiencing L-dopa withdrawal, while producing no exacerbation of memory and paired associates learning deficits. Future studies will aim to define those regions of the frontostriatal system responsible for these dopamine dependent effects on cognition and to extend the results to include longitudinal studies of PD patients beginning to receive medication. Finally, we hope to be able to pursue the possibility of doubly dissociable effects on cognitive function in PD, dependent upon deficiencies in different neurotransmitter systems.

Acknowledgements. This work was supported by a Programme Grant from the Wellcome Trust to T.W. Robbins, B.J. Everitt and B.J. Sahakian. We thank Prof. P. Rabbitt for allowing us to enlist control volunteers from the North East Age Research Panel.

\section{References}

Abas M, Sahakian BJ, Levy R (1990) Neuropsychological deficits and CT scan changes in elderly depressives. Psychol Med 20:507-520

Agid Y, Ruberg M, Dubois B, Pillon B (1987) Anatomoclinical and biochemical concepts of subcortical dementia. In: Stahl SM, Iversen SD, Goodman EC (eds) Cognitive Neurochemistry, Oxford University Press, Oxford, pp 248-271

Alexander GE, Delong MR, Strick PL (1986) Parallel organization of functionally segregated circuits linking basal ganglia and cortex. Annu Rev Neurosci 9:357-381

Berger B, Gaspar P, Verney C (1991) Dopaminergic innervation of the cerebral cortex: unexplained differences between rodents and primates. TINS 14:21-27

Björklund A, Lindvall O (1984) Dopamine containing systems in the CNS. In: Bjorklund A, Hokfelt T (eds) Handbook of chemical neuroanatomy vol 2, Classical transmitters in the CNS, Part 1. Elsevier, London, pp 55-122

Bowen FP, Kamienny MA, Burns MM, Yahr M (1975) Parkinsonism: effects of L-dopa on concept formation. Neurology 25:701-704

Brown RG, Marsden CD (1988) "Subcortical dementia". The neuropsychological evidence. Neuroscience 25:363-387

Brown RG, Marsden CD, Quinn N, Wyke MA (1984) Alterations in cognitive performance and affect-arousal state during fluctuations in motor function in Parkinson's disease. $J$ Neurol Neurosurg Psychiatry 47:454.465

Brozoski TJ, Brown RM, Rosvold HE, Goldman PS (1979) Cognitive deficit caused by regional depletion of dopamine in prefrontal cortex of rhesus monkey. Science 205:929-932

Canavan A, Passingham RE, Marsden CD, Quinn N, Wyke M, Polkey CE (1989) The performance on learning tasks of patients in the early stages of Parkinson's disease. Neuropsychologia 27:141-156

Cools AR, van der Berken JHL, Horstink MWI, van Spaendonck KPM, Berger HJC (1984) Cognitive and motor shifting aptitude disorder in Parkinson's disease. J Neurol Neurosurg Psychiatry $47: 443-453$

Cooper J, Sagar H, Jordan N, Harvey NS, Sullivan E (1991) Cognitive impairment in early, untreated Parkinson's disease and its relationship to motor disability. Brain 114:2095-2122

Divac I, Rosvold HE, Szwarcbart MK (1967) Behavioral effects of selective ablation of the caudate nucleus. J Comp Physiol Psychol $63: 184-190$

Downes JJ, Roberts AC, Sahakian BJ, Evenden JL, Morris RG, Robbins TW (1989) Impaired extra-dimensional shift performance in medicated and unmedicated Parkinson's disease: evidence for a specific attentional dysfunction. Neuropsychologia $27: 1329-1343$

El-Awar M, Becker JT, Hammond KM, Nebes RD, Boller F (1987) Learning deficit in Parkinson's disease: comparison with Alzheimer's disease and normal aging. Arch Neurol 44:180-184

Flowers KA, Robertson C (1985) The effect of Parkinson's disease on the ability to maintain a mental set. J Neurol Neurosurg Psychiatry $48: 517-529$

Gotham A-M, Brown RG, Marsden CD (1988) "Frontal" cognitive functions in patients with Parkinson"s disease "on" and "off" L-dopa. Brain $111: 299-332$

Hoehn MM, Yahr M (1967) Parkinsonism: onset, progression and mortality. Neurology 17:427-442

Kendrick DC (1985) Kendrick cognitive tests for the elderly. NFER Nelson, Windsor

Lees AJ (1990) The behavioural neurology of Parkinson's disease. In: Stern G (ed) Parkinson's disease. Chapman and Hall, London, pp 389-413

Lees AJ, Smith $\mathrm{E}$ (1983) Cognitive deficits in the early stages of Parkinson's disease. Brain 106:257-270

Milner B (1964) Some effects of frontal lobectomy in man. In: Warren JM, Akert K (eds) The frontal granular cortex and behaviour. McGraw-Hill, New York, pp 313-331 
Milner B (1982) Some cognitive effects of frontal-lobe lesions in man. Philos Trans R Soc Lond B298:211-226

Mishkin M (1982) A memory system in the monkey. Philos Trans R Soc B298: 85-92

Morris RG, Downes JJ, Evenden JL, Sahakian BJ, Heald A, Robbins TW (1988) Planning and spatial working memory in Parkinson's disease. J Neurol Neurosurg Psychiatry 51:757-766

Nelson HE (1982) National adult reading test manual. NFER Nelson, Windsor

Owen AM, Downes JJ, Sahakian BJ, Polkey CE, Robbins TW (1990a) Planning and spatial working memory following frontal lobe lesions in man. Neuropsychologia 28:1021-1034

Owen AM, Galton M, Leigh PNL, Marsden CD, Quinn N, Sahakian BJ, Summers B, Robbins TW (1990b) Planning and spatial working memory in Parkinson's disease. Soc Neurosci Abstr $16: 453-10$

Owen AM, Roberts AC, Polkey C, Sahakian BJ, Robbins TW (1991) Extra-dimensional versus intradimensional set shifting performance following frontal lobe excisions, temporal lobe excisions or amygdalo-hippocampectomy in man. Neuropsychologia 29:993-1006

Petrides M (1985) Deficits on conditional associative-learning tasks after frontal and temporal lobe lesions in man. Neuropsycholo. gia 23:601-614
Roberts AC, Everitt BJ, Robbins TW (1988) Extra- and intradimensional shifts in man and marmoset. Q J Exp Psychol 40B: $321-342$

Sahakian BJ, Morris RG, Evenden JL, Heald A, Levy R, Philpot M, Robbins TW (1988) A comparative study of visuospatial memory and learning in Alzheimer type dementia and Parkinson's disease. Brain 111:695-718

Sahakian BJ, Downes JJ, Eagger S, Evenden JL, Levy R, Philpot M, Roberts AC, Robbins TW (1990) Sparing of attentional relative to mnemonic function in a subgroup of patients with dementia of the Alzheimer type. Neuropsychologia $28: 1197-1213$

Shallice T (1982) Specific impairments of planning. Philos Trans R Soc Lond B298: 199-209

Weingartner H, Burns S, Debel R, Lewitt PA (1984) Cognitive impairment in Parkinson's disease: distinguishing between effort-demanding and autonomic processes. Psychiat Res $11: 223-235$

Winer BJ (1971) Statistical principles in experimental design, 2nd edn. McGraw Hill, New York

Yahr M (1990) Principles of medical treatment. In: Stern G (ed) Parkinson's disease. Chapman and Hall, London, pp 495-508 